\title{
PULSAR VELOCITIES
}

\author{
MATTHEW BAILES \\ Australia Telescope National Facility, CSIRO \\ P.O. Box 76 \\ Epping, N.S.W. \\ 2121 Australia
}

\begin{abstract}
Lyne \& Lorimer (1994) recently demonstrated that revisions to the pulsar distance scale, coupled with new interferometric measurements of pulsar proper motions and a better treatment of selection effects, indicate that typical pulsar velocities are of the order $450 \mathrm{~km} \mathrm{~s}^{-1}$. This is between a factor of 2-4 greater than most estimates made over the last decade. This paper looks at the implications of these higher velocities for the various theories about their origin. An extremely simple argument is used to place a fairly rigid upper limit for the rate at which neutron star pairs merge of $10^{-5} \mathrm{yr}^{-1}$ in the Galaxy. It appears inevitable that an extremely large fraction of binaries containing neutron stars coalesce during the commonenvelope stage of massive binary evolution.
\end{abstract}

\section{Introduction}

Early work on the observed pulsar distribution led Gunn \& Ostriker (1970) to propose that pulsars are high-velocity objects, with typical velocities of $\sim 100 \mathrm{~km} \mathrm{~s}^{-1}$. In the 1980s many pulsars had their velocities measured using interferometric (Lyne, Anderson \& Salter 1982) or scintillation techniques (Cordes 1986). These measurements confirmed that pulsars often possess velocities of several hundred $\mathrm{km} \mathrm{s}^{-1}$, much greater than that of their progenitors, the OB stars, whose typical velocities are a few tens of $\mathrm{km} \mathrm{s}^{-1}$. How do pulsars achieve such high velocities? 


\section{The Observational Data}

For many years, the Lyne et al. (1982) interferometric study provided the only reasonable sample of pulsar proper motions and hence velocities. The study measured the proper motions or meaningful upper limits for 26 pulsars. However, as much information was derived from the direction of the pulsar proper motion vectors as from their magnitude, and these data provided the basis for a number of fundamental conclusions concerning radio pulsars, their evolution and kinematics. These are listed below:

- Radio pulsars have large transverse velocities with an rms of $\sim 170 \mathrm{~km} \mathrm{~s}^{-1}$, and a mean of $130 \mathrm{~km} \mathrm{~s}^{-1}$.

- Most pulsars are leaving the Galactic plane.

- The "kinetic ages" of pulsars are usually less than the spin-down age, indicating that pulsar magnetic fields decay.

- A correlation exists between the transverse velocity and magnetic field strength (Anderson \& Lyne 1983).

- There is no preferential orientation of the pulsar spin and velocity vectors.

Cordes (1986) used the scintillation properties of pulsars to estimate the velocities of 69 pulsars. The mean velocity he obtained for pulsars was closer to $100 \mathrm{~km} \mathrm{~s}^{-1}$. In Fig. 1, the estimated distribution of pulsar velocities at several epochs are shown. In an important paper, Harrison \& Lyne (1993) demonstrated that the difference between the mean velocities of pulsars with velocities measured by interferometric and scintillation techniques could be understood if the scattering screen was not assumed to be midway between the pulsar and the Sun, but rather considerably closer to the Galactic plane, as might be expected. This explains the difference between the velocity distribution obtained from the two techniques.

In the late 1980 s there were three independent pulsar proper-motion surveys commenced (Bailes et al. 1990; Fomalont et al. 1992; Harrison, Lyne \& Anderson 1993), more than doubling the number of pulsars with known proper motions; this was largely due to the Harrison et al. (1993) survey. Pulsars for such proper-motion surveys were generally chosen on the basis of distance and flux, the former allowing measurement of proper motion on a reasonable time scale, and the latter providing adequate signalto-noise ratio. These more recent surveys have measured the proper motions of pulsars more distant from the Sun, and consequently further from the Galactic plane than the earlier surveys. Restricting the sample to local objects in effect excludes old, high-velocity pulsars. Compensation for this increases the mean speed by a factor of 1.2. This effect was first recognised by Cordes (1986) and recently quantified by Lyne \& Lorimer (1994). 
The latest pulsar distance model of Taylor \& Cordes (1993) is much more elaborate than that of Lyne, Manchester \& Taylor (1985). The need for a new distance model was emphasized by the discovery of the globular cluster pulsars, for which the existing model significantly underestimated distances. The new model also attempts to address the enhanced free electron density associated with spiral arms, and the tendency for the old model to underestimate the distances to nearby pulsars. Lyne \& Lorimer have demonstrated that adopting the new pulsar distance model increased the mean velocity of pulsars by a factor of 1.6.

The two effects mentioned above account for the increase in mean transverse velocity shown in Fig. 1. Lyne \& Lorimer (1994) argue that the observed pulsar velocity distribution is biased by the inclusion of old, lowvelocity pulsars. Old, high-velocity pulsars do not generally appear in pulsar proper motion surveys as they reside far from the Galactic plane. By removing all pulsars with ages $>3 \mathrm{Myr}$ we end up with the "unbiased" transverse velocity distribution shown in Fig. 1d. This distribution has a mean of $370 \mathrm{~km} \mathrm{~s}^{-1}$, almost a factor of four greater than the mean implied by the Cordes (1986) study.

How believable is this new velocity distribution? It is disturbing to see the assumed velocity distribution change so drastically over just a few years. I believe that there are several factors which might be biasing the velocity distribution toward higher velocities. One is that any simple distance model will inevitably overestimate the distances to some pulsars and underestimate the distance to others. It may be that no simple distance model is appropriate to our Galaxy, and that several pulsars have their distances and hence velocities (derived from proper motions), greatly overestimated. Another factor is that proper motions measured are the vector addition of the true proper motion, the measurement error and any systematic errors. The vector nature of these quantities usually makes the derived proper motion greater than the true proper motion. Nevertheless, unless the new distance model is severely in error, it appears that radio pulsars often have large velocities, and that when we take into account the unknown radial component of velocity, a mean of $400 \mathrm{~km} \mathrm{~s}^{-1}$ is not unreasonable. Frail (these proceedings), using the displacement of pulsars associated with supernova remnants, obtains a similar mean velocity to that derived by Lyne \& Lorimer. 

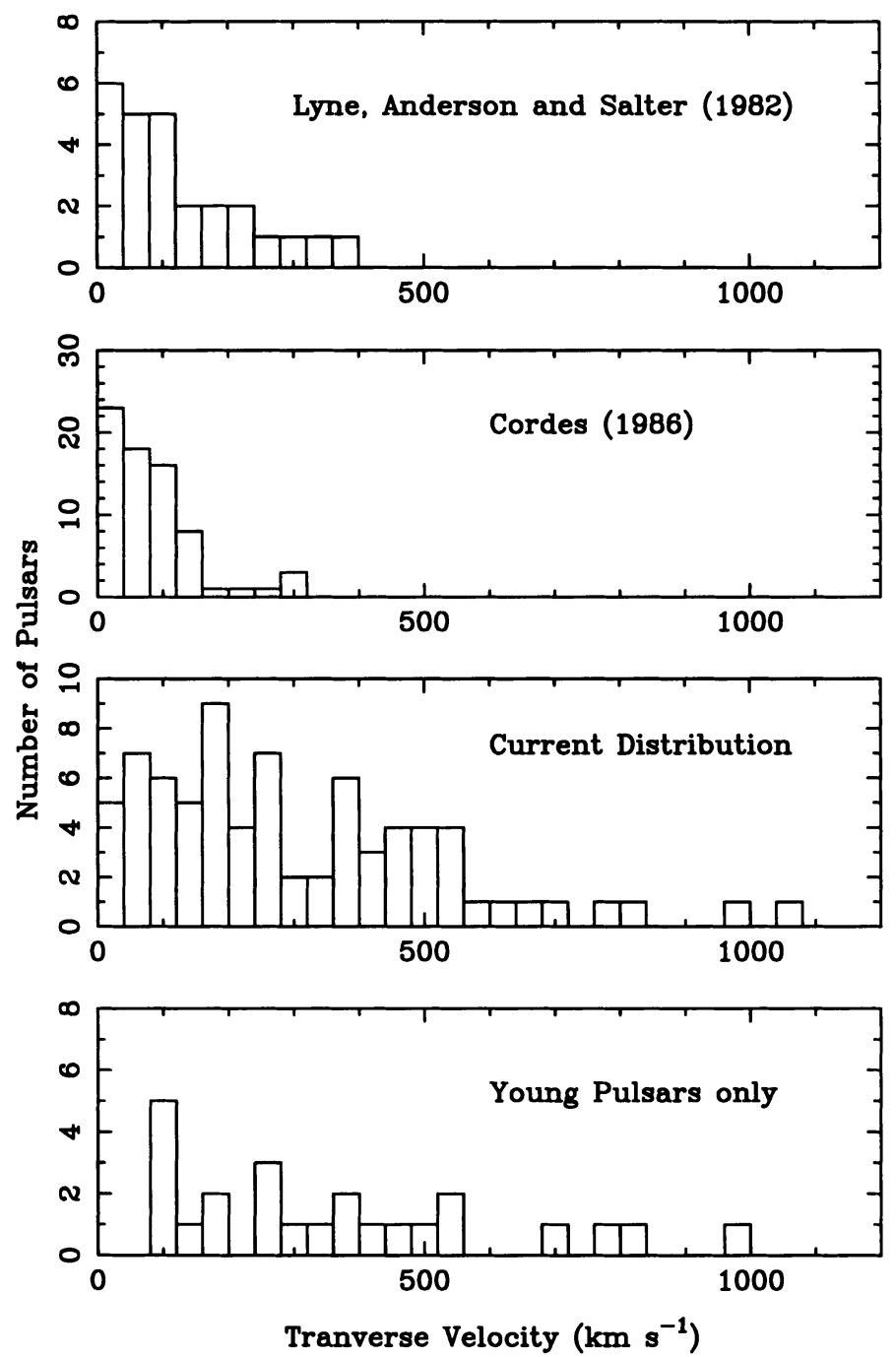

Figure 1. The changing distribution of pulsar transverse velocities. From the top: the distribution derived from the interferometric observations of Lyne, Anderson \& Salter (1982), the scintillation velocities of Cordes (1986), the most recent distribution using the latest pulsar distance model, and the distribution if we restrict the sample to pulsars younger than $3 \mathrm{Myr}$. 


\section{The Origin of the Velocities}

It has been proposed that the large velocities of radio pulsars occur because neutron stars often appear in tight binaries which disrupt during the supernova explosion that produces the pulsar. The velocities are therefore just a product of the pre-supernova conditions. This elegant idea, put forward by Gott, Gunn \& Ostriker (1970), was largely championed by V. Radhakrishnan and co-workers in the 1980 s. Unfortunately, the vast majority of pulsars are not members of binary systems, and hence what constitutes a typical pre-supernova binary is hard to determine.

In an early paper, before the appearance of much reasonable proper motion data, Radhakrishnan \& Srinivasan (1981) recognised the important role that a companion could play in the final appearance of a pulsar. They used evolutionary arguments to identify pulsars which may have been "processed" in a binary system. The binary pulsar PSR $1913+16$ is generally accepted to have accreted matter from a companion, and this is thought to explain its weaker than average magnetic field strength and shorter spin period. Radhakrishnan \& Srinivasan used some fundamental physical arguments to demonstrate that the first-born pulsars in massive binaries should have space velocities greater than that of the second-born pulsars if there were no additional "kicks" imparted to the pulsars at birth. PSR 1913+16 was used as an example of a first-born pulsar. They identified two other pulsars, PSR 1804-08 and PSR 1541-52, as weak-field, and hence the first-born pulsars in binaries which disrupted at the time of the second supernova explosion. They bravely predicted that these pulsars should have large proper motions.

Ultimately it was shown that weak-field pulsars appeared to have lower velocities than strong-field ones (Anderson \& Lyne 1983), and even though neither PSR 1804-08 nor 1541-52 had meaningful upper limits on their velocities, the appearance of the velocity-magnetic moment correlation caused extensive revision to be made to the binary break-up model. Radhakrishnan (1984) abandoned the idea that massive binaries produce a fast, weak-field pulsar and a slow, strong-field pulsar. Instead, he introduced the idea that some binaries containing a neutron star and a main-sequence star become "completely tidally disrupted", leaving behind a solitary neutron star, with a low field strength and velocity. In this revised model, massive binaries produced two high-velocity pulsars, and lower-mass binaries formed slower, weak-field pulsars. This could therefore reproduce the correlation observed between velocity and magnetic moment.

Dewey \& Cordes (1987) made very detailed simulations of the pulsar population to examine whether the observed pulsar velocity distribution could be explained without the use of some acceleration mechanism or 
"asymmetric kick" such as that proposed by Shklovskii (1970). Their conclusion was that it could not, because there were too many low-velocity pulsars in any synthesized population compared with that of the real pulsars. They chose the velocity distribution well fitted by Cordes' scintillation measurements, with a mean velocity of only $100 \mathrm{~km} \mathrm{~s}^{-1}$. Now that we believe that this underestimates the true velocities of pulsars by a factor of $\sim 4$, the conclusions of Dewey \& Cordes are strongly reinforced. The real issue here though is what fraction of pulsars are assumed to come from single stars. Dewey \& Cordes assumed that the fraction was close to $50 \%$. It is trivial to show that $50 \%$ of pulsars do not have velocities as small as the OB stars. If we hypothesize that a much larger fraction of pulsars comes from binaries than simple measurements of the binary fraction of OB stars suggest, then the argument against the binary break-up model is not so simple. More compelling evidence that pulsars receive some sort of velocity kick comes from recent evidence that the spin axis is misaligned with the orbital angular-momentum axis in PSR 1913+16 (Weisberg, Romani \& Taylor 1989), and that some young pulsars have extremely high velocities of the order of $1000 \mathrm{~km} \mathrm{~s}^{-1}$ (Cordes, Romani \& Lundgren 1993; Frail \& Kulkarni 1991). Radhakrishnan \& Shukre (1985) have shown that it is difficult to accelerate a pulsar using binaries to velocities much greater than $500 \mathrm{~km} \mathrm{~s}^{-1}$ without the system becoming too compact before the final supernova explosion.

Since the paper of Dewey \& Cordes (1987), most of the effort in the pulsar velocity debate has gone into explaining the correlation between velocity and magnetic moment. Bailes (1989) determined the velocities of the firstand second-born neutron stars in binaries similar to those Dewey \& Cordes had assumed, to demonstrate that, even with kicks, tight binaries would produce an anti-correlation between velocity and magnetic moment and far too many binary pulsars. My conclusion at the time was that most binaries disrupt whilst still wide, probably by avoiding the common-envelope phase. Bhattacharya \& Van den Heuvel (1992) suggested an elegant solution to the problem in which they postulated that all pulsars received kicks, but many binaries remain bound after the first supernova explosion and coalesce during the common-envelope phase. This model has many attractive features. First, it avoids the production of too many binary pulsars. Second, the common-envelope phase does not have to be avoided. Third, it is still possible to get low-velocity pulsars which have accreted matter and may have slightly weaker fields as a result, producing a very weak correlation between velocity and magnetic field strength, as is observed.

Recently, Camilo, Nice \& Taylor (1993) reported the discovery of a distant $60 \mathrm{~ms}$ pulsar, with remarkably similar spin period and field strength to that of PSR $1913+16$. The $z$-height of this pulsar is large $(\sim 1 \mathrm{kpc})$ and it 
seems very likely that it has a large velocity to have attained such a height. It appears therefore, that with the discovery of a weak-field, high-velocity pulsar, the original prediction of Radhakrishnan \& Shukre (1985) has been finally fulfilled. It is fascinating to see how the correlation led Radhakrishnan (1984) to propose the coalescence idea to preserve the binary break-up model only to have it incorporated into the rival model.

An offset dipole could cause a radio pulsar to accelerate to speeds of up to several $100 \mathrm{~km} \mathrm{~s}^{-1}$ from asymmetric radiation of the spin-down energy (Harrison \& Tademaru 1975). The spin vector and proper motion vector should be aligned if this mechanism is responsible for pulsar acceleration. Anderson \& Lyne (1983) dismissed the rocket mechanism as there was no correlation between the spin and velocity vectors. In the light of our newfound doubts about field decay (Bailes 1989; Bhattacharya et al. 1992), the true ages of radio pulsars can easily exceed the gravitational oscillation period of the disk. Thus, when comparing the alignment between the spin axes and proper motions of pulsars, old pulsars in which the Galactic potential has had time to act should be excluded. A revised study, which omits all old pulsars could be the final word on whether the rocket mechanism could be the pulsar acceleration mechanism.

One interesting thing to come out of the recent velocity measurements is that the apparent correlation between velocity and magnetic field strength has weakened. Although it is still statistically significant, the scatter in the data is enormous. Lorimer (1994) has shown that there is about a $7 \%$ chance that it is totally spurious. A small class of low-field, low-velocity pulsars could entirely explain the observed "correlation".

\section{The Paucity of Neutron Star Binaries}

Although there are now a few dozen binary pulsars known, most of these contain millisecond pulsars. There are only three convincing neutron star pairs in the Galactic disk, PSRs $1913+16,1534+12$ and $2303+46$. It is possible that PSR 1820-11 contains two neutron stars but this is hard to establish. It seems incredible that such a large fraction of massive stars are members of binaries and yet so few neutron star pairs exist. If half of all massive stars are members of binaries, then over $99 \%$ of systems must either disrupt or coalesce to avoid the production of too many binary pulsars. With two supernova explosions, one might imagine that disruption probabilities of $90 \%$ per explosion might suffice, but even with large kick velocities such probabilities are hard to attain. A further complication is that it is vital to retain enough massive binaries to explain the incidence of massive $\mathrm{X}$-ray binaries.

The problem with kicks is that the fraction of pulsars we expect to be 
members of binaries becomes very large when the kick velocity is of similar magnitude to the relative orbital velocity of any pre-supernova binary (Bailes 1989). This is because many pulsars get kicked into bound orbits. The only way to avoid this is to keep binaries very wide, or to have them coalesce during the common-envelope phase.

We only know of two systems which are capable of coalescing in less than a Hubble time, PSRs $1913+16$ and $1534+12$. In both cases the spin periods and field strengths of the pulsars place them far from the general pulsar population on the magnetic-field, spin-period diagram - at the location we would expect from the effects of mass transfer. It is with some confidence therefore that we identify them as the first-born pulsars in the binary, which was subsequently spun up during the giant phase of the companion. If we believe the unbiased transverse-velocity distribution shown in Fig. 1, and there are no "kicks", almost all radio pulsars must originate from very tight binaries prior to the second explosion. Since the only binary pulsars which would have been tight enough to produce two high-velocity neutron stars have, at most, a few single pulsars similar to them in the observed sample, we can produce the following argument against symmetric supernova explosions:

If almost all pulsars emerge from tight binaries similar to the progenitors of PSRs $1913+16$ and $1534+12$ and over 99\% of them disrupt to achieve the correct fraction of binary pulsars, why is the single pulsar population not inundated with high-velocity pulsars with similar spin periods and magnetic fields to the two pulsars mentioned above?

The only satisfactory solution is that pre-supernova binaries that lead to $1913+16$-like systems must be extremely rare. I conclude that close binaries at the time of the second supernova must be avoided by the complete spiralin of neutron stars into their massive companions. $R$. Taam reaches similar conclusions from entirely different reasoning in these proceedings.

How rare are $1913+16$-like systems and their progenitors? The answer is crucial, because these systems are the only guaranteed source of gravitational waves strong enough to be detected by the next generation of detectors. Several authors have tried to answer this question by extrapolating the population of the two known neutron star-neutron star binaries, first to the rest of the Galaxy, and then to the universe (Phinney 1991; Narayan, Piran \& Shemi 1991). These studies obtain "conservative" values for the coalescence rate of neutron star pairs of $10^{-6} \mathrm{yr}^{-1}$ for the Galaxy. Both PSR 1913+16 and PSR 1534+12 have weak fields and long radio lifetimes. Recently Van den Heuvel (1992) suggested that these rates are far too conservative and should be multiplied by a factor of 100 to take into account all of the neutron star binaries with much shorter radio lifetimes than the recycled pulsars we see. He claims that the coalescence rate should be 
nearer $10^{-4} \mathrm{yr}^{-1}$. The implication is that we can observe only one in every hundred neutron star pairs.

The easiest way to counter such an enormous population of invisible neutron star pairs is to consider, not the recycled pulsar in the binary, but rather the second-born or "normal" pulsar. Fortunately, the second-born pulsar has no peculiar characteristics, and should simply resemble the 650 or so other normal pulsars we know of. The statistics of these are much more certain than those of the two known neutron star binaries. Lorimer et al. (1993) estimate the birth rate of normal pulsars to be $1 / 125-1 / 250 \mathrm{yr}^{-1}$. A salient fact exists. The birth rate of normal pulsars in neutron star binary pairs must equal the birth rate of recycled pulsars in similar systems. We do not know of a single "normal" pulsar in a neutron star binary that will coalesce in a Hubble time. Therefore the birth rate of relativistic binary pulsars is less than one in 650 times the maximum pulsar birth rate, or

$$
\frac{1}{650} \times \frac{1}{125} \sim 10^{-5} \mathrm{yr}^{-1} \text {. }
$$

This is a factor of ten less than the birth rate suggested by Van den Heuvel and is based upon 650 objects, not two.

If we take the above conservative upper limit on the birth rate of coalescing neutron star binaries and combine it with the conservative lower limits of previous workers, the coalescence rate $R$ is starting to be reasonably well constrained with $10^{-6} \mathrm{yr}^{-1}<R<10^{-5} \mathrm{yr}^{-1}$. However, the earlier estimates were based upon the fact that a certain fraction of the Galaxy had been searched for such objects and only two had been found. Since then, the amount of the Galaxy that has been extensively surveyed for short-period pulsars has increased dramatically, with no more relativistic binaries discovered, despite a five-fold increase in the number of millisecond pulsars. The first-order correction to the birth rate estimates of neutron star pairs is to decrease the birth rate of relativistic binaries by a factor of five to $\sim 210^{-7} \mathrm{yr}^{-1}$.

We conclude that, although the birth rate of relativistic binary pulsars in the Galaxy is uncertain, it is probably between $210^{-7}$ and $10^{-5} \mathrm{yr}^{-1}$.

\section{Conclusions}

Radio pulsars have large space velocities which are probably obtained as the result of asymmetry in the explosion which produced the pulsar. It is difficult to disrupt binaries routinely where the kick is of the same order as the relative velocity of the binary constituents, and the paucity of neutron star binaries therefore dictates that very few binaries can resemble the progenitors of PSRs $1913+16$ and $1534+12$ before the final explosion. If massive binaries with neutron star companions usually coalesce during 
the common-envelope phase of their evolution the small fraction of binary pulsars can be understood. The end-product of such evolution is open to debate, but may produce solitary millisecond pulsars, slow weak-field pulsars or completely invisible neutron stars. The coalescence rate of neutron star binary pairs can be constrained by the complete lack of any "normal" pulsars known in such systems.

Acknowledgments. I thank V. Radhakrishnan, E.P.J. van den Heuvel, S.R. Kulkarni, A.G. Lyne, R.J. Dewey, J. Cordes and R.N. Manchester for many stimulating discussions over the years on this subject.

\section{References}

Anderson, B. \& Lyne, A.G. 1983, Nat 303, 597

Bailes, M. 1989, ApJ 342, 917

Bailes, M. et al. 1990, MNRAS 247, 322

Bhattacharya, D. \& Van den Heuvel, E.P.J. 1991, Phys. Reports 203, 1

Bhattacharya, D. et al. 1992, A\&A 254, 198

Camilo, F., Nice, D.J. \& Taylor, J.H. 1993, ApJ 412, L37

Cordes, J.M. 1986, ApJ 311, 183

Cordes, J.M., Romani, R.W. \& Lundgren, S.C. 1993, Nat 362, 133

Dewey, R.J. \& Cordes, J.M. 1987, ApJ 321, 780

Fomalont, E.B. et al. 1992, MNRAS 258, 497

Frail, D.A. \& Kulkarni, S.R. 1991, Nat 352, 785

Gott, J.R., Gunn, J.E. \& Ostriker, J.P. 1970, ApJ 160, L91

Gunn, J.E. \& Ostriker, J.P. 1970, ApJ 160, 979

Harrison, E.R. \& Tademaru, E. 1975, ApJ 201, 447

Harrison, P.A. \& Lyne, A.G., 1993 MNRAS, 265, 778

Harrison, P.A., Lyne, A.G. \& Anderson, B. 1993, MNRAS 261, 113

Lorimer, D.R. 1994, PhD thesis, The University of Manchester

Lorimer, D.R. et al. 1993, MNRAS 263, 403

Lyne, A.G. \& Lorimer, D.R. 1994, Nat 369, 127

Lyne, A.G., Anderson, B. \& Salter, M.J. 1982, MNRAS 201, 503

Lyne, A.G., Manchester, R.N. \& Taylor, J.H. 1985, MNRAS 213, 613

Narayan, R., Piran, T. \& Shemi, A. 1991, ApJ 379, L17

Phinney, E.S. 1991, ApJ 380, L17

Radhakrishnan, V. 1984, in Millisecond Pulsars, S.P. Reynolds \& D.R. Stinebring (Eds.), NRAO (Green Bank), p. 130

Radhakrishnan, V. \& Shukre, C.S. 1985, in Supernovae, Their Progenitors and Remnants, G. Srinivasan \& V. Radhakrishnan (Eds.), Indian Academy of Sciences (Bangalore), p. 155

Radhakrishnan, V. \& Srinivasan, G. 1981, in Proc. 2nd Asian-Pacific Regional Meeting of the IAU, B. Hidayat \& M.W. Feast (Eds.), Tira Pustaka (Jakarta), p. 423

Shklovskii, I.S. 1970, Astr. Zh. 46, 715

Taylor, J.H. \& Cordes, J.M. 1993, ApJ 411, 674

Van den Heuvel, E.P.J. 1992, in X-ray Binaries and Recycled Pulsars, E.P.J. van den Heuvel \& S.A. Rappaport (Eds.), Kluwer (Dordrecht), p. 233

Weisberg, J.M., Romani, R.W. \& Taylor, J.H. 1989, ApJ 347, 1030 


\section{Discussion}

J. van Paradijs: Nick White and I (1995, ApJ, submitted) have recently calculated the galactic distribution of LMXB for several evolutionary scenarios, both with and without kick velocities (over and above that expected from sudden mass loss). We find that this extra kick is necessary to account for the distance of LMXB from the galactic plane; the Lyne and Lorimer (1994) velocity distribution - taken as kick velocity distribution given a reasonable agreement with the observed LMXB distribution.

M. Bailes: There are a large number of assumptions one needs to make in order to follow the evolution of the system from post-explosion to LMXB, I suspect that within the parameter space there is room for kicks from Lyne and Lorimer (1994), to say a factor of two lower than this.

V. Lipunov: What is the $3 \sigma$ accuracy of the mean velocity?

M. Bailes: The Lyne and Lorimer (1994) estimate is $450 \pm 90 \mathrm{~km} \mathrm{~s}^{-1}$ where $90 \mathrm{~km} \mathrm{~s}^{-1}$ is the statistical uncertainty only $(1 \sigma)$. If you wanted to say that the distance scale is wrong by $30 \%$ you could increase this $1 \sigma$ uncertainty to $\sim 120 \mathrm{~km} \mathrm{~s}^{-1}$. Extending this to $3 \sigma$ gives an enormous value of $450 \pm 360 \mathrm{~km} \mathrm{~s}^{-1}$. 